\title{
En pacientes con sospecha de apendicitis, la tomografía computada mejora el diagnóstico y baja los costos
}

Effect of computed tomography of the apendix on treatment of patients and use of hospital resources. Rao P, James T, Rhea M, et al. N Engl J Med 1998; 338:141-6

\section{Objetivo}

Evaluar la eficacia diagnóstica de la tomografía computada (TC) en la apendicitis aguda, y su impacto en la utilización de los recursos hospitalarios.

\section{Diseño}

Estudio de corte transversal

\section{Lugar}

Guardia del Massachusets General Hospital, Boston, EE.UU.

\section{Pacientes}

Se estudiaron 100 pacientes que ingresaron en forma consecutiva a la guardia, con el diagnóstico presuntivo de apendicitis aguda. La población estudiada constaba de 57 mujeres y 43 hombres, entre 6 y 75 años de edad.

\section{Descripción del test y el test de referencia}

Al ingreso a la guardia, se le realizaba a los pacientes una evaluación clínica y de laboratorio. El cirujano de guardia estimaba la probabilidad de apendicitis como: definida ( 80 a 100\%), probable (60 a 79\%), equivoca (40 a 59\%) o posible (20 a 39\%). Posteriormente se realizaba una TC Localizada helicoidal con contraste, que era interpretada por uno de los tres radiólogos de emergencia en un lapso no mayor a 1 hora, estos estimaban la probabilidad de apendicitis como: definitivamente $\mathrm{Si}$, probablemente $\mathrm{Si}$, equivocadamente $\mathrm{Si}$, probablemente No o definitivamente No. El diagnóstico se realizaba en la cirugía, por examen anátomo-patológico o durante un seguimiento clínico de por lo menos dos meses.

\section{Medición de resultados principales}

Los diagnósticos finales se agruparon en: apendicitis, condiciones específicas alternativas o dolor abdominal no específico. El potencial cambio en el cuidado de los pacientes luego de la intervención diagnóstica de la TC, se midió comparando el tratamiento planeado inicialmen- te (internación en observación ó cirugía de urgencia), con el tratamiento recibido posteriormente.

Los cambios en la utilización de recursos hospitalarios se determinaron de la misma forma, comparando los costos del tratamiento planificado antes de realizar la TC con los del recibido después de realizada la TC. Se calcularon previamente los costos de la extracción de un apéndice normal, la internación en observación y la realización de la TC. El número de apendectomías innecesarias ahorradas por el procedimiento diagnóstico, se multiplicó por el costo de remover un apéndice normal. El número de días de observación ahorrados se multiplicó por el costo de días de internación hospitalaria. La diferencia del costo global se calculó de restar el costo de las 100 TC realizadas con el ahorro resultante de incorporar la TC a la sistemática de estudio de los pacientes con sospecha de apendicitis aguda.

\section{Resultados Principales}

De los 100 pacientes evaluados, 54 tuvieron apendicitis y 46 no la tuvieron. La TC fue positiva en 54 pacientes, de los cuales 53 tuvieron apendicitis; y negativa en 46 , de los cuales 45 no tuvieron apendicitis (Sensibilidad* $98 \%$, Especificidad* $98 \%$, VPP* 98\%, VPN* 98\%). La estimación clínica catalogó 67 casos como apendicitis definitiva ó probable, de los cuales 20 no fueron apendicitis. La estimación radiológica consideró como apendicitis definitiva ó probable a 53 pacientes, de los cuales uno no fue apendicitis. Los cambios en el tratamiento por los hallazgos tomográficos muestran que se previnieron 13 apendicectomías innecesarias con un ahorro de 47.281 dólares, y días de internación en 50 pacientes, con un ahorro de 20.250 dólares. El costo de las 100 TC helicoidal, fue de 22800 dólares, por lo cual el ahorro total fue de 44.731 dólares.

\section{Conclusiones}

La utilización de TC en pacientes con sospecha de apendicitis mejora el rédito diagnóstico, el cuidado de los pacientes y disminuye los costos.

\section{COMENTARIO}

El presente es el estudio que con mayor rigor evalúa el impacto de la TC en el diagnóstico diferencial de apendicitis. Es importante destacar que el estudio utiliza la TC helicoidal, método aún poco disponible en nuestro país, y sus resultados no son directamente trasladables a la TC convencional. En lo referente a lo metodológico, debe analizarse un aspecto importante. El estudio no estratifica a los pacientes por edad, sólo menciona que se incorporaron pacientes entre 6 a 75 años, sin precisar la media de la edad. Esta tiene una importancia decisiva en establecer el diagnóstico correcto. En pacientes pediátricos el principal diagnóstico diferencial es la adenitis mesentérica, de resolución no quirúrgica, pero con una imagen radiológica claramente diferenciable de la apendicitis ${ }^{1-2}$. En el presente estudio 18 pacientes tuvieron este diagnóstico final, no queda claro que se debiera incluirlos sin diferenciarlos. En el grupo geriátrico la patología prevalente es la neoplásica y vascular, y frente a la toma de decisiones en un paciente mayor de 60 años con abdomen agudo, sin causas médicas reconocidas, la exploración quirúrgica es la regla. Es muy difícil que una TC cambie la decisión, y , en este caso, el diagnóstico de apendicitis ó no es irrelevante ${ }^{3}$.

Dejando de lado estas poblaciones, los resultados obtenidos son contundentes, la eficacia diagnóstica es del $98 \%$, y supera a la evaluación clínica. De acuerdo al estudio, aún en aquellos casos donde la probabilidad clínica de una apendicitis esté entre el 70 y el 100\%, debe confirmarse el diagnóstico con una TC. La razón se explica claramente por los resultados, ya que de 57 casos en que el cirujano interpretó apendicitis, sólo se confirmaron 37 (67\%) y de 43 casos considerados como poco probables, 16 la tuvieron.

Otro aspecto a considerar es la extrapolación de los resultados a nuestro medio, ya que la TC, debe hacerse y evaluarse rápidamente. En un medio como el nuestro con quirófanos atestados de urgencias graves, requeriría de una planificación importante. Por otra parte la impresión subjetiva (perdón para los amantes de la evidencia), de aquellos que tuvieron años de experiencia en guardias es que la tasa de error diagnóstico que presenta el autor de este artículo es un poco elevada.

También deberían considerarse otros estudios alternativos a la TC como la ecografía, con reportes de eficacia diagnóstica similares a los reportados aquí, y la laparoscopía que es el método de elección en los abdómenes agudos de fuerte presunción ginecológica ${ }^{4-5}$.

Con respecto al costo-beneficio, sin dudas el saldo de ahorro de 400 dólares por paciente es significativo. Sin embargo el estudio es demasiado pequeño para evaluar que sucedería frente a un imponderable como por ejemplo un cáncer insospechado. Por otra parte los costos en estas latitudes son diferentes, en relación fundamentalmente al día cama y a los procedimientos diagnósticos.

*Ver Glosario

Dr. Pablo Argibay

Servicio de Cirugía General. Hospital Italiano de Buenos Aires.

Referencias

1. Reynold S. Missed appendicitis in a pediatric emergency department. Pediatr Emerg Care 1993; 9:1-3

2. Rao PM, Rhea JT, Novelline RA. CT diagnosis of mesenteric adenitis. Radiology 1997: 202 (1): 145-9

3. Mccallion J, Canning GP, Knigth RV. Acute apendicitis in the elderly: a five years retrospective study. Age ageing 1987; 16: 256-60

4. Kalle T, Mc Donald EB, Prantl F. Sonography of acute appendicitis in children: 7 years experience. Pediatr Radiol 1998 Mar, 28(3): 147-51

5. Tytgat SH, Baaker XL, Butzelaar RM. Laparoscopic evaluation of patients with suspected appendicitis. Surg Endosc 1998; 12(7): 918-20 